Pacific Journal of Mathematic 


\section{BAER RINGS AND QUASI-CONTINUOUS RINGS HAVE A MDSN}

\section{G. F. BirKenMEIER}

The notion of a direct summand of a ring containing the set of nilpotents in some "dense" way has been considered by Y. Utumi, L. Jeremy, C. Faith, and G. F. Birkenmeier. Several types of rings including right selfinjective rings, commutative FPF rings, and rings which are a direct sum of indecomposable right ideals have been shown to have a MDSN (i.e., the minimal direct summand containing the nilpotent elements). In this paper, the class of rings which have a MDSN is enlarged to include quasiBaer rings and right quasi-continuous rings. Also, several known results are generalized. Specifically, the following results are proved: (Theorem 3) Let $R$ be a ring in which each right annihilator of a reduced (i.e., no nonzero nilpotent elements) right ideal is essential in an idempotent generated right ideal. Then $R=A \oplus B$ where $B$ is the MDSN and an essential extension of $N_{t}$ (i.e., the ideal generated by the nilpotent elements of index two), and $A$ is a reduced right ideal of $R$ which is also an abelian Baer ring. (Corollary 6) Let $R$ be an $A W^{*}$-algebra. Then $R=$ $A \oplus B$ where $A$ is a commutative $A W^{*}$-algebra, and $B$ is the MDSN of $R$ and $B$ is an $A W^{*}$-algebra which is a rational extension of $N_{t}$. Furthermore, $A$ contains all reduced ideals of $R$. (Theorem 12) Let $R$ be a ring such that each reduced right ideal is essential in an idempotent generated right ideal. Then $R=A \oplus B$ where $B$ is the densely nil MDSN, and $A$ is both a reduced quasi-continuous right ideal of $R$ and a right quasi-continuous abelian Baer ring.

From [8 \& 14], a ring $R$ is (quasi-) Baer if it has unity and the right annihilator of every (right ideal) nonempty subset of $R$ is generated by an idempotent. A Baer ring is abelian if all its idempotents are central. The following examples will give some indication of the wide application of these rings: (i) von Neumann algebras, such as the algebra of all bounded operators on a Hilbert space, are Baer rings [2, pp. $21 \& 24]$; (ii) the commutative $C^{*}$ algebra $C(T)$ of continuous complex valued functions on a Stonian space is a Baer ring [2, p. 40]; (iii) the ring of all endomorphisms of an abelian group $G$ with $G=D \oplus E$, where $D \neq 0$ is torsion-free divisible and $E$ is elementary, is a Baer ring [16]; (iv) any right self-injective von Neumann regular ring is Baer [17, p. 253]; (v) any prime ring is quasi-Baer; (vi) since a $n \times n$ matrix ring over a 
quasi-Baer ring is quasi-Baer [15], the $n \times n(n>1)$ matrix ring over a non-Prufer commutative domain is a prime quasi-Baer ring which is not a Baer ring [14, p. 17]; (vii) since a $n \times n$ lower triangular matrix ring over a quasi-Baer ring is a quasi-Baer ring [15], the $n \times n(n>1)$ lower triangular matrix ring over a domain, which is not a division ring, is quasi-Baer but not Baer [14, p. 16]; (viii) semiprime right FPF rings are quasi-Baer [10, p. 168]. The examples show that the class of Baer rings does not contain the class of prime rings and is not closed under extensions to matrix rings or triangular matrix rings. However the notion of a quasiBaer ring overcomes these shortfalls.

Throughout this paper, all rings are associative; $R$ denotes a ring with unity; $N(X)$ is the set of nilpotent elements of $X(N$ will be used when $X=R$ ) and $N_{t}$ is the ideal generated by the nilpotent elements of index two. The word ideal will mean a two-sided ideal unless it is preceded by the words left or right. A reduced ring is one without nonzero nilpotent elements. Note that in a reduced ring all idempotents are central. A right ideal $X$ of $R$ is densely nil (DN) if either $X=0$; or $X \neq 0$ and every nonzero right ideal of $R$ which is contained in $X$ has nonzero intersection with $N$. Equivalently, a right ideal $X$ of $R$ is densely nil if either $X=0$; or $X \neq 0$ and for every nonzero $x \in X$ there exists $r \in R$ such that $x r \neq 0$ but $(x r)^{2}=0$. From [3], the minimal direct summand (idempotent generated right ideal) containing the nilpotent elements $M D S N$ is a semicompletely prime ideal (i.e., if $x^{n} \in \operatorname{MDSN} \Rightarrow x \in \mathrm{MDSN}$ ) which equals the intersection of all idempotent generated right ideals containing the set of nilpotent elements of the ring. Also any nonzero direct summand of the MDSN has a nonzero nilpotent element, and the MDSN contains both the right singular ideal of $R$ and the generalized nil radical $\left[\begin{array}{lll}1 & \& & 3\end{array}\right]$. There are nonreduced rings which do not have a MDSN [3, Example 2.6]. The complement of the MDSN is both a maximal reduced idempotent generated right ideal which is unique up to isomorphism and a reduced ring with unity.

LEMMA 1. Let $R$ be reduced. Then $R$ is a quasi-Baer ring if and only if $R$ is an abelian Baer ring. In particular, a commutative quasi-Baer ring is a reduced Baer ring.

Proof. In a reduced ring every right annihilator is an ideal. Hence the right annihilator of any subset will equal the right annihilator of the right ideal generated by the subset. Therefore $R$ is quasi-Baer if and only if $R$ is Baer. Commutative quasi-Baer rings are reduced because a quasi-Baer ring contains no nonzero central nilpotent elements. This completes the proof. 
Lemma 2. If $X$ is an ideal of $R$ such that $X \cap N_{t} \neq 0$, then $X$ contains a nonzero nilpotent element of index two.

Proof. From [1 \& 9], we have that the generalized nil radical $N_{g}$ is hereditary and contains $N_{t}$. Thus, if $X \cap N_{t} \neq 0$ then $X \cap N_{g}(R)=$ $N_{g}(X) \neq 0$ contains a nonzero nilpotent element of index two.

A right $R$-module $B$ is an essential extension of a submodule $X$ (equivalently, $X$ is essential in $B$ ) in case for every submodule $L$ of $B, X \cap L=0$ implies $L=0$. The next theorem presents a decomposition in terms of a reduced Baer ring and the MDSN, furthermore the MDSN is "essentially" generated by the nilpotents of index two.

THEOREM 3. Let $R$ be a ring in which each right annihilator of a reduced right ideal is essential in an idempotent generated right ideal. Then $R=A \oplus B$ where $B$ is the MDSN and an essential extension of $N_{t}$, and $A$ is a reduced right ideal of $R$ which is also an abelian Baer ring.

Proof. Let $X=\bigoplus_{i \in I} e_{i} R$ be maximal among reduced direct sums of idempotent generated right ideals [3, p. 714]. Let $Y$ be the right annihilator of $X$. From [3, Prop. 1.2], $X N=0$, so $N \leqq Y$. Thus, if $Y=0$ then $R=A$. If $Y \neq 0$ then there exists $y=y^{2}$ such that $y R$ is an essential extension of $Y$. By [3, Prop. 1.2], $y R$ is an ideal. Then $Y=y R$ because $X(y R) \subseteq X \cap y R=0$ since $X$ is reduced. To show that $y R$ is the MDSN, let $0 \neq t=t^{2} \in y R$. Assume $t R \cap N=0$. By the maximality of $X,(X \oplus t R) \cap N \neq 0$. Hence there exists $x \in X$ and $c \in t R$ such that $0 \neq x+c$ and $(x+c)^{2}=0$. Thus $(x+c)^{2}=x^{2}+x c+c x+c^{2}=x^{2}+c x+c^{2}=0$. Then $x^{2}=$ $\left(-c x-c^{2}\right) \in X \cap Y=0$. Hence $x=0$ and $c=0$ because $X$ and $t R$ are reduced. Contradiction! Therefore $y R=B$ is the MDSN [3, Thrm. 1.4] and $(1-y) R=A$ is a reduced ring with unity.

Let $0 \neq s \in B$ and assume $s R \cap N_{t}=0$. Hence $(s R) N_{t}=0$. Therefore $N_{t}$ is contained in the right annihilator of $s R$. There exists $e=e^{2}$ such that $e R$ is an essential extension of the right annihilator of $s R$. Hence $N_{t} \leqq e R$. Thus $B \subseteq e R$, since $B$ is the MDSN [3, Thrm. 1.4]. But this is a contradiction because $s R$, which is reduced, cannot be contained in $e R$. Therefore $N_{t}$ is essential in $B$.

Let $D$ be a right ideal of $A$. By [3, Lem. 1.1], $D$ is a right ideal of $R$. Let $U$ be the right annihilator of $D$ in $R$. There exists $u=u^{2}$ such that $u R$ is an essential extension of $U$. Now $(1-y) U$ is the right annihilator of $D$ in $A$, and $(1-y) u R$ is an essential extension of $(1-y) U$ in $A$ with $((1-y) u)^{2}=(1-y) u$. Thus $D \cap$ $(1-y) u R=0$. Since $A$ is reduced $(1-y) u$ is a central idempotent 
in $A$. Hence $(1-y) u R$ is an ideal in $A$. Thus $D((1-y) u R) \subseteq D \cap$ $(1-y) u R=0$. Therefore $(1-y) U=(1-y) u R$. Hence $A$ is a quasi-Baer ring. By Lemma $1, A$ is an abelian Baer ring. In fact, any idempotent generated reduced right ideal of $R$ is an abelian Baer ring. This completes the proof.

From Lemma 2, it can be seen that every nonzero ideal of $R$ contained in $B$ has nonzero nilpotent elements. Hence every reduced ideal of $R$ is contained in $A$. Also, we note that commutative FPF rings (e.g., integers $\bmod 12$ ) are not necessarily quasi-Baer but satisfy the hypothesis of Theorem 3 [10, p. 168 Lem. 3A]. In fact, Theorem 3 is a generalization of the decomposition for commutative FPF rings obtained by C. Faith [10, p. 184].

Corollary 4. Let $R$ be a quasi-Baer ring. Then $R=A \oplus B$ where $B$ is the MDSN and an essential extenison of $N_{t}$, and $A$ is a reduced right ideal of $R$ which is also an abelian Baer ring.

Letting $R$ be the $2 \times 2$ lower triangular matrix ring over a domain (e.g., example vii) and applying Corollary 4, we have

$$
A=\left|\begin{array}{ll}
1 & 0 \\
0 & 0
\end{array}\right| R \text { and } B=\left|\begin{array}{ll}
0 & 0 \\
0 & 1
\end{array}\right| R \text {. }
$$

A right $R$-module $B$ is a rational extension of a submodule $X$ if whenever $x, y \in B$ with $x \neq 0$, there exists an element $r \in R$ such that $x r \neq 0$ and $y r \in X$. Note that if $B$ is a rational extension of $X$ then $B$ is an essential extension of $X$. A MDSN ring is a ring which equals its MDSN.

COROllary 5. Let $R$ be a semiprime (quasi-)Baer ring. Then $R=A \oplus B$ (ring direct sum) where $B$ is a MDSN (quasi-)Baer ring which is a rational extension of $N_{t}$, and $A$ is a reduced abelian Baer ring.

Proof. From Corollary $4, R=A \oplus B$ where $B$ is the MDSN. Let $B=b R$ where $b=b^{2}$ and $A=(1-b) R$. Then $b R(1-b)=0$ because $R$ is semiprime. Since $1-b$ is a unity for $A$, it follows that $1-b$ is central in $R$. Hence $A$ and $B$ are rings with unity. Let $0 \neq x \in B$. Then $x R \cap N_{t} \neq 0$ since $N_{t}$ is essential in $B$. Now $x N_{t} \neq 0$ because $R$ is semiprime. Let $r \in N_{t}$ such that $x r \neq 0$. Then, for any $y \in B, y r \in N_{t}$. Hence $B$ is a rational extension of $N_{t}$. This completes the proof.

Corollaries 4 and 5 generalize Proposition 5.7 of $[17$, p. 255] and Rangaswamy's decomposition of a von Neumann regular Baer ring which is the endomorphism ring of an abelian group [16]. Further- 
more, according to Kaplansky's classification of Baer rings [14], types II and III and semiprime type $I_{\text {inf }}$ are MDSN rings. Hence reduced Baer rings are type $I_{\text {fin }}$.

Three important classes of semiprime quasi-Baer rings are the class of semiprime right FPF rings [10, p. 168], the class of von Newmann regular Baer rings (e.g., Examples (i), (iii) and (iv)) and the class of Baer *-rings (i.e., a ring with involution *, such that the right annihilator of any subset is generated by a projection $[14, \mathrm{p}$. 27]). A useful subclass of Baer *-rings are the $A W^{*}$-algebras (i.e., a $C^{*}$-algebra which is Baer ${ }^{*}[2$, p. 21]). Examples (i) and (ii) are $A W^{*}$-algebras [2, pp. 21, 24, 40]. From Lemma 1 and [14, p. 10], we note that an $A W^{*}$-algebra is commutative if and only if it is reduced. Thus for $A W^{*}$-algebras we have

Corollary 6. Let $R$ be an $A W^{*}$-algebra. Then $R=A \oplus B$ (ring direct sum) where $A$ is a commutative $A W^{*}$-algebra and $B$ is a MDSN $A W^{*}$-algebra which is a rational extension of $N_{t}$. Furthermore, $A$ contains all reduced ideals of $R$.

We remark that a quasi-Baer ring which is a rational extension of $N_{t}$ is not necessarily $D N$. Let $R$ be a $2 \times 2$ matrix ring over a domain which is not a left Ore domain. By [15], $R$ is a prime quasi-Baer ring; and from [5] $R$ is MDSN. Hence, by Corollary $5, R$ is a rational extension of $N_{t}$. However, $R$ is not $D N$ [5].

The next lemma and theorem show that under mild finiteness conditions (e.g., no infinite direct sums of index two nilpotent left ideals or no infinite direct sums of reduced subrings with unity) a nonsemiprime (quasi-)Baer ring can be decomposed in terms of a reduced Baer ring, a nilpotent ring and a (quasi-) Baer MDSN ring.

LEMMA 7. Let $R$ be a ring which is neither reduced nor MDSN and in which at least one of the following conditions holds:

(i) $R$ is a direct sum of indecomposable right ideals;

(ii) $R$ has no infinite sets of orthogonal idempotents $\left\{e_{1}, e_{2}, \cdots\right\}$ such that each of $e_{i} R e_{i}$ is reduced;

(iii) $R$ has no infinite direct sums of nilpotent left ideals of the form $b R a$ where $a$ and $b$ are orthogonal idempotents with $a R a$ reduced and the ring eRe has a MDSN for every nonzero idempotent $e \in R$.

Then there exists a positive integer $n$ such that for each $k=1, \cdots, n$ there is an idempotent $b_{k}$ where $R b_{k}=b_{k} R b_{k}$, a reduced ring $S_{k}$ with unity, and a left ideal $X_{k}$ of $R$ such that $X_{k}^{2}=0$. Also, $R b_{k-1}$ is ring isomorphic to 


$$
\left|\begin{array}{cc}
S_{k} & 0 \\
X_{k} & R b_{k}
\end{array}\right|
$$

with $b_{0}=1$ and $X_{k} \oplus R b_{k}$ (left ideal direct sum) is the MDSN of $R b_{k-1}$. Consequently, $R=S \oplus X \oplus R b_{n}$ (additive group direct sum) where $S=\bigoplus_{k=1}^{n} S_{k}$ (ring direct sum) is a reduced ring, $X=\bigoplus_{k=1}^{n} X_{k}$ is a nilpotent left ideal of $R$, and $R b_{n}$ is a reduced ring with unity or a MDSN ring with unity.

Proof. The proof of the lemma using condition (i) is in [4]. Suppose that condition (ii) holds. Then by [3, Thrm. 2.4] $R$ has a MDSN. Hence there exists $e=e^{2}$ such that $R=e R \oplus(1-e) R$ where $e \neq 0$ and $e \neq 1$, and $e R=e R e$ is a reduced ring with unity and $(1-e) R$ is the MDSN of $R$. Let $b_{1}=1-e, X_{1}=b_{1}$ Re, and $S_{1}=e R$. By [4, Lem. 1] $R=S_{1} \oplus X_{1} \oplus R b_{1}$ (additive group direct sum), $X_{1}$ is a left ideal of $R$ such that $X_{1}^{2}=0, R b_{1}=b_{1} R b_{1}$ is a ring with unity, and $X_{1} \oplus R b_{1}=b_{1} R$ (left ideal direct sum) where $b_{1} R$ is the MDSN of $R$. If $R b_{1}$ is reduced or if $R b_{1}$ is MDSN then we are finished; otherwise we will continue the decomposition with $R_{1}=$ $b_{1} R b_{1}=R b_{1}$. Observe if $a=a^{2} \in R_{1}$ then $a R_{1} a=a\left(b_{1} R b_{1}\right) a=a R a$ since $b_{1}$ is the unity of $R_{1}$. Thus $R_{1}$ has no infinite sets of orthogonal idempotents $\left\{a_{1}, a_{2}, \cdots\right\}$ such that each $a_{i} R_{1} a_{i}$ is reduced. Again by [3, Thrm. 2.4], $R_{1}$ has a MDSN. Hence there exists $e_{1}=e_{1}^{2}$ such that $e_{1} R_{1}=e_{1} R_{1} e_{1}=e_{1} R e_{1}$ is a reduced ring with unity and $\left(b_{1}-e_{1}\right) R_{1}$ is the MDSN of $R_{1}$. Let $b_{2}=b_{1}-e_{1}, S_{2}=e_{1} R_{1}$, and $X_{2}=b_{2} R_{1} e_{1}=$ $b_{2} R e_{1}$. Note $\left\{e, e_{1}, b_{2}\right\}$ is a set of orthogonal idempotents of $R$. From [4, Lem. 1] $b_{2} R_{1} b_{2}=R_{1} b_{2}=R b_{2}=b_{2} R b_{2}$ is a ring with unity and thus $R=S_{1} \oplus X_{1} \oplus S_{2} \oplus X_{2} \oplus R b_{2}$ (additive group direct sum) where $S_{1} \oplus S_{2}$ is a ring direct sum of reduced rings with unity. Since $b_{1} R$ is an ideal in $R$, it can be shown that $r=e r e+b_{1} r e+b_{1} r b_{1}$ for $r \in R$. Thus $r X_{2}=\left(b_{1} r b_{1}\right) X_{2} \subseteq X_{2}$ because $X_{2}$ is a left ideal of $R_{1}$ [4, Lem. 1]. Therefore $X_{2}$ is a left ideal of $R$. Hence $X_{1} \oplus X_{2}$ is a nilpotent left ideal of $R$. Also $X_{2} \oplus R b_{2}$ (left ideal direct sum) is the MDSN of $R_{1}$. If $R b_{2}$ is reduced or if $R b_{2}$ is MDSN, then we are finished; otherwise, we will continue with $R_{2}=R b_{2}$. Since $\left\{e, e_{1}\right\}$ is a set of orthogonal idempotents such that $e R e$ and $e_{1} R e_{1}$ are reduced, one can see that the above procedure will terminate after, say, $n$ steps. Thus it follows that $R$ has the desired group direct sum decomposition. The triangular matrix characterization for $R b_{k-1}$ follows from [4, Lem. 1].

The proof of the theorem using condition (iii) is similar to the above proof except that "the procedure will terminate after, say, $n$ steps because there can be only finitely many $X_{i}$." This completes the proof. 
Corollary 8. Let $R=S \oplus X \oplus R b_{n}$ as in Lemma 7. Then $N(R)=\left\{k \in R \mid k=x+y\right.$ where $x \in X$ and $\left.y \in N\left(R b_{n}\right)\right\}$, and $N(R)$ is an ideal of $R$ if and only if $N\left(R b_{n}\right)$ is a left ideal of $R b_{n}$. Furthermore, if $N(R)$ is an ideal of $R$ then $N(R)=X \oplus N\left(R b_{n}\right)$ (direct sum of left ideals of $R$ ).

Proof. From repeated use of [4, Lem. 3], $N(R)=\{k \in R \mid k=x+y$ where $x \in X$ and $\left.y \in N\left(R b_{n}\right)\right\}$. Suppose $N(R)$ is an ideal of $R$. $N\left(R b_{n}\right)=$ $N(R) \cap R b_{n}$. Thus $N\left(R b_{n}\right)$ is a left ideal of $R$. Hence $N\left(R b_{n}\right)$ is a left ideal of $R b_{n}$, in fact $N\left(R b_{n}\right)$ is an ideal of $R b_{n}$; and $N(R)=X \oplus$ $N\left(R b_{n}\right)$ (direct sum of left ideals of $R$ ).

Conversely, assume $N\left(R b_{n}\right)$ is a left ideal of $R b_{n}$. From the triangular representation of $R b_{k}$, it follows that a left ideal of $R b_{n}$ is also a left ideal of $R$. Hence $N\left(R b_{n}\right)$ is a left ideal of $R$. Thus $N(R)=X \oplus N\left(R b_{n}\right)$ (direct sum of left ideals of $R$ ). Consequently $N(R)$ is a left ideal of $R$, hence $N(R)$ is an ideal of $R$. This completes the proof.

We note that if $R b_{n}$ is reduced then $N(R)=X$ is a ideal of $R$.

TheOREM 9. Let $R$ be a (quasi-)Baer ring which is neither reduced nor MDSN and in which at least one of the following conditions holds:

(i) $R$ is a direct sum of indecomposable right ideals;

(ii) $R$ has no infinite sets of orthogonal idempotents $\left\{e_{1}, e_{2}, \cdots\right\}$ such that each $e_{i} R_{i}$ is reduced;

(iii) $R$ has no infinite direct sums of nilpotent left ideals of the form $b R a$ where $a$ and $b$ are orthogonal idempotents with aRa reduced.

Then there exists a positive integer $n$ such that for each $k=1, \cdots, n$ there is an idempotent $b_{k}$ where $R b_{k}=b_{k} R b_{k}$, a reduced Baer ring $S_{k}$, and a left ideal $X_{k}$ of $R$ such that $X_{k}^{2}=0$. Also $R b_{k-1}$ is ring isomorphic to

$$
\left|\begin{array}{cc}
S_{k} & 0 \\
X_{k} & R b_{k}
\end{array}\right|
$$

with $b_{0}=1$ and $X_{k} \oplus R b_{k}$ (left ideal direct sum) is the MDSN of $R b_{k-1}$. Consequently, $R=S \oplus X \oplus R b_{n}$ (additive group direct sum) where $S=\bigoplus_{k=1}^{n} S_{k}$ (ring direct sum) is a reduced Baer ring, $X=$ $\bigoplus_{k=1}^{n} X_{k}$ is a nilpotent left ideal of $R$, and $R b_{n}$ is a reduced Baer. ring or $R b_{n}$ is a MDSN (quasi-)Baer ring. Furthermore, $N(R)$ is an ideal of $R$ if and only if $N\left(R b_{n}\right)$ is a left ideal of $R b_{n}$.

Proof. The proof follows from Lemma 7, Corollary 4, Corollary 
8 and the fact that if $e=e^{2}$ and $R$ is (quasi-)Baer then $e R e$ is (quasi-) Baer [8] and [14, p. 6].

By observing that an indecomposable reduced Baer ring is a domain, it follows that if $R$ satisfies condition (i) or (ii) of Theorem 9 , then each $S_{k}$ is a ring direct sum of domains; consequently $S$ is a ring direct sum of domains.

From [13], a right $R$-module $M$ is quasi-continuous (also known as $\pi$-injective [11]) if it satisfies the following two conditions:

(i) each submodule of $M$ is essential in a direct summand of $M$.

(ii) if $P$ and $Q$ are direct summands of $M$ such that $P \cap Q=0$, then $P \oplus Q$ is a direct summand of $M$.

From [7], a right $R$-module $M$ is a CS module if and only if each complement submodule of $M$ is a direct summand of $M$, equivalently each submodule of $M$ is essential in a direct summand of $M$ (i.e., condition (i) in the definition above). A ring is right (quasicontinuous) $C S$ if it is (quasi-continuous) $C S$ as a right $R$-module [6]. Right self-injective rings and products of right Ore domains are right quasi-continuous [13 \& 19]. A $n \times n(n>1)$ lower triangular matrix ring over a field is a right $C S$ ring which is not a right quasi-continuous ring.

Proposition 10. Let $R$ be a semiprime ring such that the right annihilator of every ideal is essential in an idempotent generated right ideal of $R$. Then $R$ is quasi-Baer.

Proof. Let $X$ be an ideal of $R$ and $Y$ is the right annihilator of $X$. Then $X \cap Y=0$ since $R$ is semiprime. Let $e R$ be an essential extension of $Y$ with $e=e^{2}$. Hence $X \cap e R=0$. Now $(X e R)^{2}=$ $(X e R)(X e R)=X(e R X) e R=0$. Thus $X(e R)=0$. Therefore $Y=e R$. By [8, Lem. 1], $R$ is quasi-Baer.

CoROllaRY 11. Let $R$ be a semiprime ring. If $R$ is a CS ring or a right quasi-continuous ring, then $R$ is a quasi-Baer ring.

Corollary 11 has no converse since a domain which is not a right Ore domain is quasi-Baer, but such a domain is not a right CS ring. Also the semiprime condition is necessary since the integers $\bmod 4$ form a quasi-continuous ring which is not quasi-Baer.

The next theorem and corollaries generalize several results [3, Thrm. 3.9], [11, Thrm. 1.15], and [13, Prop. 5.2 \& Prop. 5.5].

THEOREM 12. Let $R$ be a ring such that each reduced right ideal is essential in an idempotent generated right ideal. Then $R=A \oplus B$ 
where $B$ is the densely nil MDSN, and $A$ is both a reduced quasicontinuous right ideal of $R$ and a right quasi-continuous abelian Baer ring.

Proof. By Zorn's lemma there exists a maximal reduced right ideal $K$. From [13, Lem. 5.1], any right ideal which is an essential extension of $K$ is also reduced. From the maximality of $K, a R=K$ where $a=a^{2}$. By [3, Prop. $1.2 \&$ Prop. 1.7], $A=K$ and $B=(1-a) R$ is the densely nil MDSN. Since every right ideal of $A$ is a right ideal of $R$ [3, Lem. 1.1] we need only show that $A$ is a right quasicontinuous ring. We note that part (ii) of the definition of a quasicontinuous module is satisfied since every idempotent is central in a reduced ring. To show part (i) of the definition of a quasi-continuous module, let $X$ be a nonzero right ideal of $A$. Then there exists $e=e^{2}$ such that $X$ is essential in $e R$ as an $R$-module. Now $X \subseteq a e R$ and $(a e)^{2}=a e \in A$. Let $0 \neq a e r \in a e R$. Then $e r \neq 0$, hence there exists $s \in R$ such that $0 \neq e r s \in X \subseteq A$. Thus ers $=$ aers $=(a e r)(a s)$ since $a$ is a unity for $A$. Therefore $X$ is essential in $a e R$ as an $A$ module and as an $R$-module. From Lemma 1 and Corollary 11, $A$ is a reduced abelian Baer ring. This completes the proof.

COROLlaRY 13. Let $R$ be a right (quasi-continuous) CS ring. Then $R=A \oplus B$ where $B$ is the (quasi-continuous) CS densely nil MDSN, and $A$ is both a quasi-continuous reduced right ideal of $R$ and a right quasi-continuous abelian Baer ring

From the proof of Theorem 12, one can see that if the reduced right ideals of a ring are essential in idempotent generated right ideals then every idempotent generated reduced right ideal of $R$ is a quasi-continuous reduced abelian Baer ring. Furthermore, A contains every reduced ideal of $R$ since $B$ is $D N$. Also, any condition on $R$ (such as semiprime) which forces $(1-a) R a=0$ will make the decomposition a ring decomposition. From Corollary 11 and Corollary 13 we have:

COROLLARY 14. Let $R$ be a semiprime right (quasi-continuous) $C S$ ring. Then $R=A \oplus B$ where $A$ is a right quasi-continuous reduced abelian Baer ring, and $B$ is a (quasi-continuous) CS densely nil quasi-Baer ring.

Any $n \times n(n>1)$ lower triangular matrix ring over the integers provides an example for Theorem 9 and Theorem 12 which is a quasi-Baer ring but not a right $C S$ ring [6, p. 73]. Example (iv) satisfies the hypothesis of Corollary 14. Also, from [12, Thrm. 2.3] 
and [13, p. 219], any strongly modular Baer*-ring is a semiprime quasi-continuous ring and thus satisfies the hypotheses of Corollaries 5 and 14 . In particular, any finite $A W^{*}$-algebra (e.g., example (ii) is a strongly modular Baer*-ring [12, p. 14].

\section{REFERENCES}

1. V. A. Andrunakievic and Jm. M. Rjabukin, Rings without nilpotent elements, and completely simple ideals, Soviet Math. Dokl., 9 (1968), 565-568.

2. S. K. Berberian, Baer*-rings, Grundlehren math. Wiss., Band 195, Springer Verlag, New York, 1972.

3. G. F. Birkenmeier, Self-injective rings and the minimal direct summand containing the nilpotents, Comm. in Alg., 4 (8) (1976), 705-721.

4. - Indecomposable decompositions and the minimal direct summand containing the nilpotents, Proc. Amer. Math. Soc., 73 (1979), 11-14.

5. G. F. Birkenmeier and R. P. Tucci, Does every right ideal of a matrix ring contain a nilpotent element?, Amer. Math. Monthly, 84 (1977), 631-633.

6. A. W. Chatters and C. R. Hajarnavis, Rings in which every complement right ideal is a direct summand, Quart. J. Math. Oxford, (2), 28 (1977), 61-80.

7. A. W. Chatters and S. M. Khuri, Endomorphism rings of modules over nonsingular CS rings, to appear.

8. W. E. Clark, Twisted matrix units semigroup algebras, Duke Math. J., 34 (1967), $417-424$.

9. N. J. Divinsky, Rings and Radicals, Mathematical Expositions 14, University of Toronto Press, Toronto, 1965.

10. C. Faith., Injective quotient rings of commutative rings, in Module Theory, Springer Lecture Notes No. 700, Berlin, Springer-Verlag, 1979, 151-203.

11. V. K. Goel and S. K. Jain, $\pi$-injective modules and rings whose cyclics are -injective, Comm. Algebra, 6 (1978), no. 1, 59-73.

12. D. Handelman, Coordinatization applied to finite Baer*-rings, Trans. Amer. Math. Soc., 235 (1978), 1-34.

13. L. Jeremy, Modules et anneaux quasi-continuous, Canad. Math. Bull., 17 (2) (1974), 217-228.

14. I. Kaplansky, Rings of Operators, Mathematics Lecture Note Series, W. A. Benjamin, New York, 1968.

15. A. Pollingher and A. Zaks, On Baer and quasi-Baer rings, Duke Math. J., 37 (1970), 127-138.

16. K. M. Rangaswamy, Regular and Baer rings, Proc. Amer. Math. Soc., 42 (1974), $354-358$.

17. B. Stenstrom, Rings of Quotients, Grundlehren math. Wiss., Band 217, SpringerVerlag, New York, 1975.

18. Y. Utumi, On continuous regular rings and semi-simple self-injective rings, Canad. J. Math., 12 (1960), 597-605.

19. - On continuous rings and self-injective rings, Trans. Amer. Math. Soc.. 118 (1965), 158-173.

Received July 10, 1979 and in revised form September 29, 1980.

Southeast Missouri State University

Cape Girardeau, MO 63701 


\section{PACIFIC JOURNAL OF MATHEMATICS}

EDITORS

DONALD BABBITT (Managing Editor)

University of California

Los Angeles, CA 90024

Hugo RossI

University of Utah

Salt Lake City, UT 84112

C. C. MOORE and ANDREW OGG

University of California

Berkeley, CA 94720

\section{J. DugundjI}

Department of Mathematics

University of Southern California

Los Angeles, CA 90007

R. FINN and J. MILGRAM

Stanford University

Stanford, CA 94305

\section{ASSOCIATE EDITORS}
R. ARENS
E. F. BECKENBACH
B. H. NEUManN
F. WOLF
K. YoSHIDA

\section{SUPPORTING INSTITUTIONS}

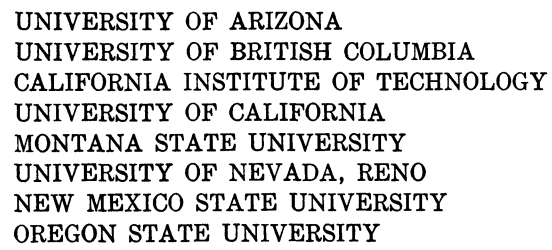

UNIVERSITY OF ARIZONA

UNIVERSITY OF BRITISH COLUMBIA

CALIFORNIA INSTITUTE OF TECHNOLOGY

UNIVERSITY OF CALIFORNIA

MONTANA STATE UNIVERSITY

UNIVERSITY OF NEVADA, RENO

NEW MEXICO STATE UNIVERSITY

OREGON STATE UNIVERSITY

\author{
UNIVERSITY OF OREGON \\ UNIVERSITY OF SOUTHERN CALIFORNIA \\ STANFORD UNIVERSITY \\ UNIVERSITY OF HAWAII \\ UNIVERSITY OF TOKYO \\ UNIVERSITY OF UTAH \\ WASHINGTON STATE UNIVERSITY \\ UNIVERSITY OF WASHINGTON
}

The Supporting Institutions listed above contribute to the cost of publication of this Journal, but they are not owners or publishers and have no responsibility for its content or policies.

Mathematical papers intended for publication in the Pacific Journal of Mathematics should be in typed form or offset-reproduced, (not dittoed), double spaced with large margins. Please do not use built up fractions in the text of the manuscript. However, you may use them in the displayed equations. Underline Greek letters in red, German in green, and script in blue. The first paragraph or two must be capable of being used separately as a synopsis of the entire paper. Please propose a heading for the odd numbered pages of less than 35 characters. Manuscripts, in triplicate, may be sent to any one of the editors. Please classify according to the scheme of Math. Reviews, Index to Vol. 39. Supply name and address of author to whom proofs should be sent. All other communications should be addressed to the managing editor, or Elaine Barth, University of California, Los Angeles, California, 90024.

50 reprints to each author are provided free for each article, only if page charges have been substantially paid. Additional copies may be obtained at cost in multiples of 50 .

The Pacific Journal of Mathematics is issued monthly as of January 1966. Regular subscription rate: $\$ 102.00$ a year (6 Vols., 12 issues). Special rate: $\$ 51.00$ a year to individual members of supporting institutions.

Subscriptions, orders for numbers issued in the last three calendar years, and changes of address shoud be sent to Pacific Journal of Mathematics, P.O. Box 969, Carmel Valley, CA 93924, U.S.A. Old back numbers obtainable from Kraus Periodicals Co., Route 100, Millwood, NY 10546.

\section{PUBLISHED BY PACIFIC JOURNAL OF MATHEMATICS, A NON-PROFIT CORPORATION}

Printed at Kokusai Bunken Insatsusha (International Academic Printing Co., Ltd.). 8-8, 3-chome, Takadanobaba, Shinjuku-ku, Tokyo 160, Japan. 


\section{Pacific Journal of Mathematics}

\section{Vol. 97, No. $2 \quad$ February, 1981}

Patrick Robert Ahern and N. V. Rao, A note on real orthogonal measures . . . . . 249

Kouhei Asano and Katsuyuki Yoshikawa, On polynomial invariants of fibered

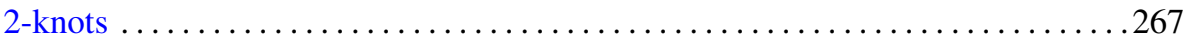

Charles A. Asmuth and Joe Repka, Tensor products for $S L_{2}(\mathscr{K})$. I.

Complementary series and the special representation

Gary Francis Birkenmeier, Baer rings and quasicontinuous rings have a

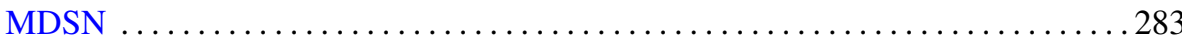

Hans-Heinrich Brungs and Günter Törner, Right chain rings and the generalized

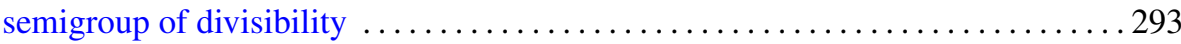

Jia-Arng Chao and Svante Janson, A note on $H^{1} q$-martingales . . . . . . . . . 307

Joseph Eugene Collison, An analogue of Kolmogorov's inequality for a class of

additive arithmetic functions

Frank Rimi DeMeyer, An action of the automorphism group of a commutative ring on its Brauer group

H. P. Dikshit and Anil Kumar, Determination of bounds similar to the Lebesgue

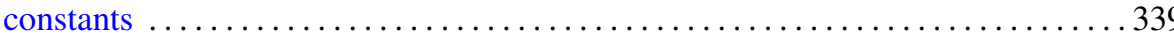

Eric Karel van Douwen, The number of subcontinua of the remainder of the plane

D. W. Dubois, Second note on Artin's solution of Hilbert's 17th problem. Order spaces

Daniel Evans Flath, A comparison of the automorphic representations of GL(3) and its twisted forms

Frederick Michael Goodman, Translation invariant closed $*$ derivations

Richard Grassl, Polynomials in denumerable indeterminates

K. F. Lai, Orders of finite algebraic groups

George Kempf, Torsion divisors on algebraic curves

Arun Kumar and D. P. Sahu, Absolute convergence fields of some triangular matrix methods

Elias Saab, On measurable projections in Banach spaces

Chao-Liang Shen, Automorphisms of dimension groups and the construction of AF algebras

Barry Simon, Pointwise domination of matrices and comparison of $\Phi_{p}$ norms

Chi-Lin Yen, A minimax inequality and its applications to variational inequalities

Stephen D. Cohen, Corrections to: "The Galois group of a polynomial with two indeterminate coefficients"

Phillip Schultz, Correction to: "The typeset and cotypeset of a rank 2 abelian group"

Pavel G. Todorov, Correction to: "New explicit formulas for the $n$th derivative of composite functions"

Douglas S. Bridges, Correction to: "On the isolation of zeroes of an analytic function" 